\title{
SECAGEM CONVECTIVA DA ACEROLA (Malphigia emarginata DC.): APLICAÇÃO DE MODELOS SEMITEÓRICOS
}

\author{
F.C. MORAES FILHO', E.L. OLIVEIRA ${ }^{2}$, E.M.M.A. NÓBREGA ${ }^{1}$, J. A. OLIVEIRA ${ }^{2}$ e R.T.P. CORREIA ${ }^{2}$ \\ ${ }^{1}$ Programa de Pós-Graduação em Engenharia Química - Universidade Federal do Rio Grande do Norte \\ ${ }^{2}$ Departamento de Engenharia Química - Universidade Federal do Rio Grande do Norte \\ fcmfilho@gmail.com - edson@eq.ufrn.br
}

Artigo submetido em julho/2012 e aceito em fevereiro/2014

\section{RESUMO}

A acerola é uma planta medicinal originária da América do Sul, muito rica em vitamina $C$, que exerce um efeito preventivo e curativo em caso de doenças infecciosas. Estima-se que $40 \%$ dos frutos da acerola são desperdiçados após a colheita. Este fato está aliado a sua alta perecibilidade e também devido a sua maior comercialização na forma in natura. O presente trabalho visou avaliar preliminarmente as curvas de secagem do resíduo da acerola (Malphigia emarginata DC.) em secador de bandejas. A partir dessas curvas foram obtidos dados de taxa de secagem e as constantes de velocidade, além do ajuste de modelos semiteóricos aos dados experimentais. As curvas de secagem foram obtidas, utilizando-se um secador de bandejas nas temperaturas de 60,70 e $80^{\circ} \mathrm{C}$ e com velocidade do ar de secagem de 4,0 e $6,0 \mathrm{~m} / \mathrm{s}$. Os dados experimentais foram ajustados aos modelos de Lewis e Henderson e Pabis, resultando, para todos os casos, em um coeficiente de regressão $\left(R^{2}\right)$ superior a 0,96 . Com base nos resultados apresentados pode-se concluir que, para as condições operacionais utilizadas, a cinética de secagem do resíduo da acerola ocorreu nos períodos de taxa constante e decrescente; a cinética foi influenciada tanto pela velocidade do ar de secagem quanto pela temperatura de operação.

PALAVRAS-CHAVE: secagem convectiva, acerola, modelagem, secador de bandejas.

\section{CONVECTIVE DRYING OF ACEROLA (Malphigia emarginata DC.): APPLICATION OF SEMITHEORETICAL MODELS}

\begin{abstract}
Acerola is a medicinal plant originated in South America, rich in vitamin $\mathrm{C}$, which exerts preventive and curative effects in cases of infectious diseases. It is estimated that $40 \%$ of acerola fruits are wasted after harvest. This fact is coupled with its high perishability and also due to their commercialization majorly made in the fresh form. This study aims to evaluate preliminarily the drying curves of the residue of acerola (Malphigia emarginata DC.) in a tray dryer. From these curves were obtained data of drying rate and velocity constant, in addition to the adjustment of experimental data with
\end{abstract}

semitheoretical models. The drying curve was obtained using a tray dryer at temperatures of 60,70 and $80^{\circ} \mathrm{C}$ and with drying air velocity of 4.0 and $6.0 \mathrm{~m} / \mathrm{s}$. Experimental data were fitted to the models of Lewis and Henderson and Pabis, resulting, in all cases, in a correlation coefficient $\left(R^{2}\right)$ greater than 0.96 . Based on the reported results, it can be concluded that, for the operating conditions used, the drying kinetics of the acerola residue occurred during constant and decreasing rate periods; kinetics was influenced by both the velocity of the drying air and the operating temperature.

KEYWORDS: convective drying, acerola, modeling, tray dryer. 


\section{INTRODUÇÃO}

O fruto da acerola, conhecida também como cereja-das-antilhas ou cereja-de-Barbados, é um fruto carnoso originário da região compreendida ao sul do México, América Central e Norte da América do Sul ((Brunini et al., 2004), cultivado em escala comercial em Porto Rico, Havaí, Jamaica e Brasil. Pertencente à família Malpighiaceae, era conhecida pelos termos Malpighia glabra L. e Malpighia punicifolia L., mas recebeu recentemente reclassificação taxonômica que resultou na determinação do nome Malphigia emarginata DC., como atual nome científico da planta (Mezadri et al., 2008).

Essa fruta tem atraído o interesse dos agricultores devido ao elevado teor de vitamina $C$, com teor de ácido ascórbico variando de 695 a 4827mg $100 \mathrm{~mL}^{-1}$ de polpa (Mezadri et al., 2006). É este atributo que tem feito essa fruta ser amplamente utilizada na alimentação humana e na fabricação de produtos farmacêuticos além de atender o mercado de polpa processada e fruta in natura. Além do ácido ascórbico, estudos recentes comprovam a existência de importantes compostos bioativos na acerola, tais compostos fenólicos e corantes naturais (Rosso e Mercadante, 2005, 2007; Rosso et al., 2008) com propriedades antioxidantes (Mezadri et al., 2008), substâncias com reconhecida ação na prevenção de doenças degenerativas, atividade biológica e manutenção da saúde (Dembitsky et al., 2011).

Parte do volume de fruto in natura é destinada para operações de beneficiamento que incluem a preparação de sucos, concentrados, néctares, além da secagem, importante operação unitária para a tecnologia de alimentos (Mezadri et al., 2008; Moreira et al., 2009). Essa técnica possibilita a diversificação dos produtos, armazenamento por longos períodos em condições ambientais e redução significativa de perdas nos períodos da safra (Jesus et al., 2003). Outras vantagens destacadas por Park (2001) são a facilidade e economia de energia na conservação do produto, relativa manutenção dos componentes aromáticos e proteção contra degradação enzimática e oxidativa. No entanto, o processo pode acarretar em redução significativa do valor nutritivo e sensorial do produto, incluindo cor, sabor e textura do alimento (Lenart, 1996; Marques et al., 2007). Dessa forma, a qualidade do produto, a melhoria das condições de operação e o custo do consumo energético têm sido temas de estudos cada vez mais frequentes entre os pesquisadores da área de engenharia de processamento de alimentos (Freire, 2011).

Pesquisadores da área de secagem de alimentos têm utilizado modelos fundamentados na teoria de difusão de líquido. Algumas considerações devem ser realizadas para a aplicação desses modelos, como por exemplo: despreza-se o encolhimento do material em processamento e os efeitos de capilaridade e de transferência de calor e massa. Também se considera que o material e o ar de secagem entram instantaneamente em equilíbrio térmico.

Alguns trabalhos sobre secagem de alimentos utilizando o secador convectivo de bandejas vêm sendo desenvolvidos no Brasil. Park (2001) avaliou a secagem de pêra em fatias da variedade Bartlett (Pyrussp.) e apresentou curvas de secagem nas temperaturas 50,60 e $70^{\circ} \mathrm{C} \mathrm{e}$ velocidades do ar de 0,5,1,0 e 1,5m/s. Através destas curvas é possível detectar o aumento da difusividade térmica com o aumento da temperatura do ar de secagem, o que reflete na diminuição das resistências internas do processo da secagem com o aumento da temperatura. 
Em sua pesquisa, Almeida et al. (2011) investigaram a cinética de secagem de frutos da acerola em secador de leito fixo com temperaturas de 50,60 e $70^{\circ} \mathrm{C}$ e velocidades do ar de 1,0 e $1,5 \mathrm{~m} / \mathrm{s}$. Com base nos resultados obtidos, os autores puderam concluir que a cinética da secagem do fruto da acerola ocorre dentro do período de taxa decrescente e que a cinética é mais influenciada pelas temperaturas de secagem e menos influenciada pela velocidade do ar.

Este trabalho teve como objetivo estudar a secagem do resíduo da acerola (Malphigia emarginata DC.) em secador de bandejas, nas condições operacionais de temperaturas de 60, 70 e $80^{\circ} \mathrm{C}$ e velocidades do ar de secagem de $4,0,5,0$ e $6,0 \mathrm{~m} / \mathrm{s}$ e ajustar os modelos de Lewis e Henderson e Pabis aos dados experimentais.

Os modelos de Lewis, Henderson e Pabis e outros têm sido amplamente utilizados, principalmente com produtos agrícolas, como sementes, grãos, frutos e em algumas espécies de plantas (Panchatiya et al., 2002 e Santos et al., 2010).

\section{METODOLOGIA}

O resíduo de acerola foi submetido ao processo de secagem para obtenção de pós, utilizando secador convectivo de bandejas, localizado no Laboratório de Energia Alternativa e Fenômenos de Transporte (LEAFT) do Departamento de Engenharia Química da UFRN.

A temperatura de trabalho foi monitorada usando-se termorresistência PT100, ligado a um controlador de temperatura que manipula a potência da resistência elétrica ao longo do experimento, mantendo a temperatura do sistema em valor pré-estabelecido (set point). No secador, o fluxo de ar é perpendicular à bandeja, com velocidade previamente estabelecida através da regulagem em válvula gaveta disposta no sistema de admissão do ar. Para monitorar o perfil de temperatura, foram colocados termopares digitais ao longo da câmara de secagem. Os termopares digitais acoplados ao secador monitoram a temperatura ambiente, temperatura das bandejas em que se encontra o produto e da saída do secador. Permite-se com isto acompanhar as variações térmicas durante o processo da secagem. Além disso, um termo-higrômetro mediu periodicamente a umidade do ar ambiente e na do ar na saída do secador e um anemômetro digital mediu a velocidade do ar na saída do secador. Na Figura 1 está ilustrado o monitoramento do processo utilizado nos experimentos de secagem do resíduo da acerola.

O resíduo agroindustrial de acerola anteriormente armazenado em freezer a temperatura de $-10^{\circ} \mathrm{C}$ foi retirado e colocado em refrigeração à temperatura de aproximadamente $5^{\circ} \mathrm{C}$ para que o descongelamento do material ocorra uniformemente. O material, então, é retirado da refrigeração até que se atinja a temperatura ambiente. A seguir, o mesmo é distribuído em bandeja de alumínio com tela perfurada confeccionada no LEAFT, e depois submetido ao processo de secagem no secador convectivo de bandejas, posicionada na altura do $4^{\circ}$ termopar (item 10 da Figura 1). Em intervalos de tempo pré-estabelecidos, a bandeja foi retirada, pesada em balança digital com precisão de 0,0001 g e recolocada no secador, a fim de obter a massa do resíduo. Este procedimento foi realizado até atingir peso constante, ou seja, quando não houve mais variação de massa, e o tempo necessário para isso foi registrado. 


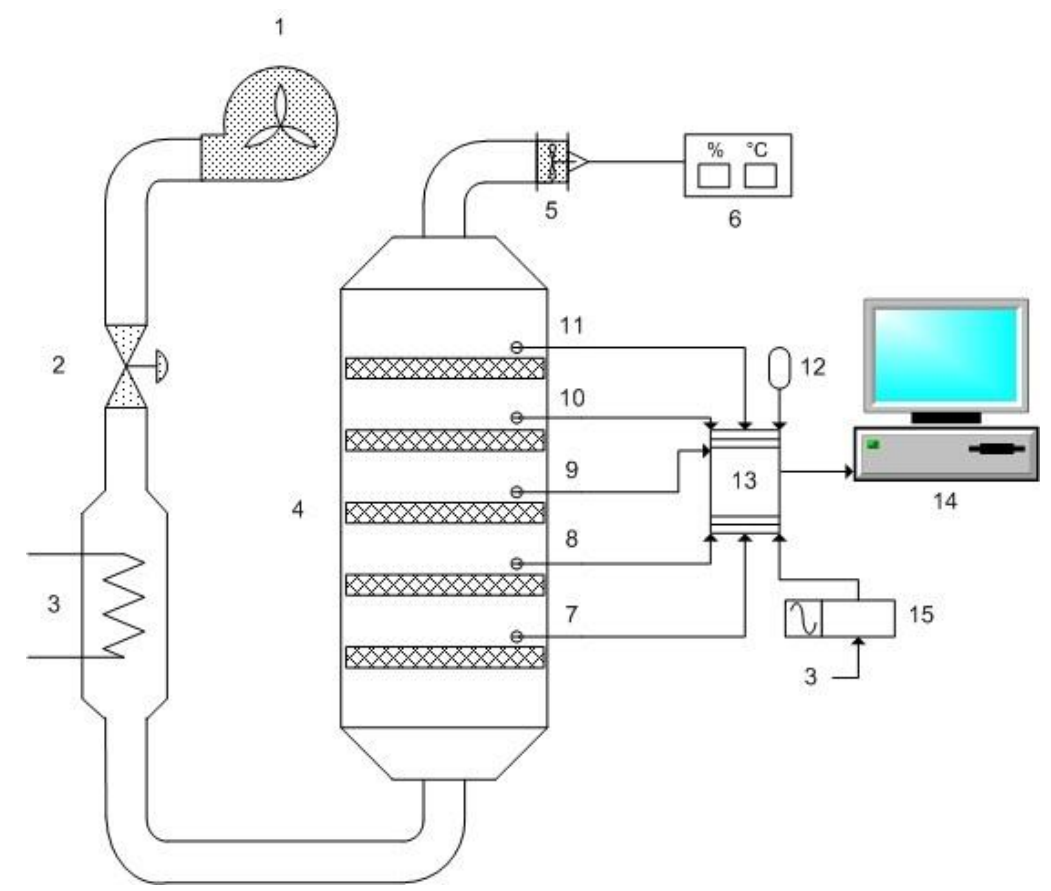

Figura 1 - Esquema de monitoramento e controle do sistema de secagem (1 - soprador; válvula de controle de vazão; 3 - trocador de calor; 4 - câmara de secagem; 5 - anemômetro; 6 - termohigrômetro; 7, 8, 9, 10, 11 termopar tipo K; 12 - sensor de temperatura ambiente, cód. LM35DT; 13 - placa de aquisição de dados; 14 computador com software SCADADRYER e 15 - relé de estado sólido).

O processo de secagem em camada delgada foi realizado a partir de Delineamento Inteiramente Casualizado (DIC) com duas repetições e com medidas em triplicata, totalizando 11 ensaios.

Os modelos de Lewis e Henderson e Pabis (H. P.), descritos, respectivamente, pelas Equações 1 e 2, foram utilizados para ajuste dos dados experimentais através de análises de regressão não linear, utilizando o software STATISTICA 7.

$\operatorname{MR}=\mathbf{e}^{\left(-\mathbf{k}_{0} \mathbf{t}\right)}$

Equação (1)

$\mathbf{M R}=\mathbf{a} \cdot \mathbf{e}^{\left(-\mathbf{k}_{0} t\right)}$

Equação (2)

Para ajuste dos modelos, foram utilizados o coeficiente de correlação $\left(R^{2}\right)$ e o parâmetro qui-quadrado reduzido $\left(\chi^{2}\right)$ calculado pela Equação 3.

$\chi^{2}=\frac{\sum_{\mathrm{i}=1}^{\mathrm{N}}\left(\mathrm{MR}_{\text {exp }, \mathrm{i}}-\mathrm{MR}_{\text {pred }, \mathrm{i}}\right)^{2}}{\mathrm{~N}-\mathrm{n}}$

Equação (3)

Onde: $\mathrm{MR}_{\text {exp,i }}$ - valores experimentais da razão de umidade para a observação, i (adimensional); $\mathrm{MR}_{\text {pred,i }}$ - valores preditos da razão de umidade para a observação, i (adimensional); $\mathrm{N}$ - número de observações; $n$ - número de parâmetros no modelo. 


\section{RESULTADOS E DISCUSSÕES}

Nesta secção são apresentados os resultados dos experimentos da secagem com resíduo da acerola em secador de bandejas a diferentes temperaturas, espessura da torta do resíduo e velocidades do ar de secagem.

Nas Figuras 2 (A), (B) e 3 (C) e (D) são apresentados os dados experimentais da secagem do resíduo da acerola, nas condições de temperatura $60^{\circ} \mathrm{C}$, velocidade do ar de secagem de 4,0 e $6,0 \mathrm{~m} / \mathrm{s}$ e espessura da torta do material de 0,50 e $0,75 \mathrm{~cm}$, respectivamente. As curvas estão apresentadas na forma adimensional pela razão (Uo/Ui) entre a umidade final e umidade inicial em função do tempo de secagem.

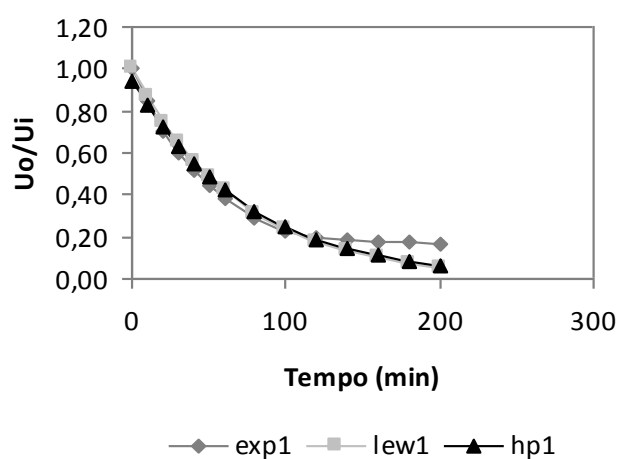

(A)

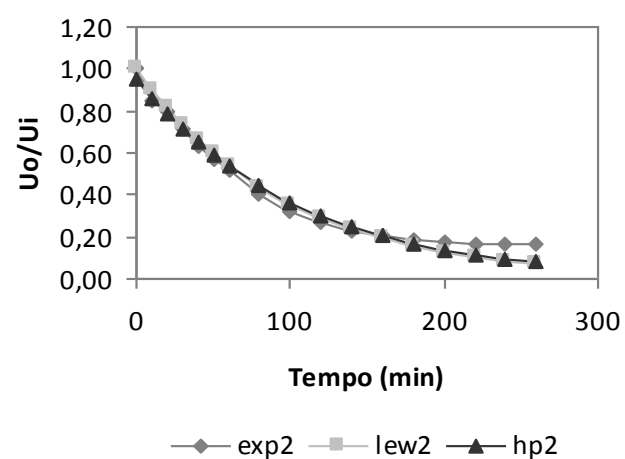

(B)

Figura 2 - Secagem do resíduo da acerola em secador de bandeja à temperatura de $60^{\circ} \mathrm{C}$ e velocidade de $4 \mathrm{~m} / \mathrm{s},(\mathrm{A})$ espessura da torta de $0,50 \mathrm{~cm}$ e (B) espessura da torta de $0,75 \mathrm{~cm}$. Legenda: (exp) valores experimentais, (lew) resultados ajustados pelo modelo de Lewis e (hp) modelo de Henderson e Pabis.

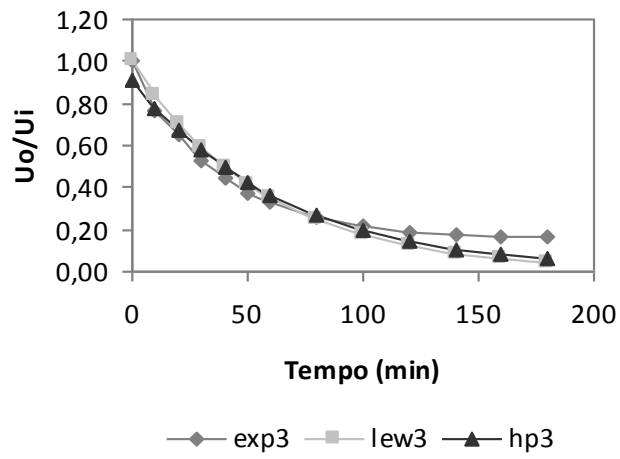

(C)

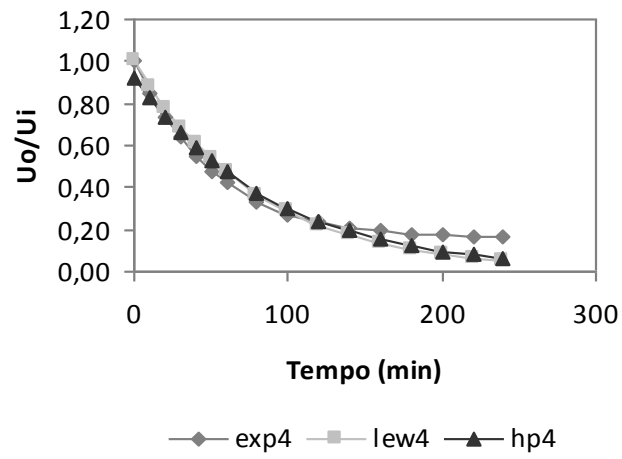

(D)

Figura 3 - Secagem do resíduo da acerola em secador de bandeja à temperatura de $60{ }^{\circ} \mathrm{C}$ e velocidade de $6 \mathrm{~m} / \mathrm{s}$, (C) espessura de $0,50 \mathrm{~cm}$ e (D) espessura de $0,75 \mathrm{~cm}$. Legenda: (exp) valores experimentais, (lew) resultados ajustados ao modelo de Lewis e (hp) modelo de Henderson e Pabis.

De maneira geral, é possível visualizar grande semelhança entre as curvas apresentadas. Por exemplo, verifica-se que para $v=4 \mathrm{~m} / \mathrm{s}, T=60^{\circ} \mathrm{C}$ e espessura $0,50 \mathrm{~cm}$ (Figura 2 (A)), o tempo de secagem foi de 200 minutos e a relação $\mathrm{Uo} / \mathrm{Ui}=0,17$ correspondente a umidade final de 0,83 b.s. Quando comparamos a condição $T=60^{\circ} \mathrm{C}$ e espessura $0,75 \mathrm{~cm}$ (Figura 2 (B)), o tempo de secagem foi $260 \mathrm{~min}$. e a relação Uo/Ui $=0,17$ correspondente a umidade final de 0,86 b.s. Da mesma maneira, $v=6 \mathrm{~m} / \mathrm{s}, \mathrm{T}=60^{\circ} \mathrm{C}$ e espessura $0,5 \mathrm{~cm}$ (Figura $3(\mathrm{C})$ ), o tempo de secagem foi de $180 \mathrm{~min}$. e a relação $\mathrm{Uo} / \mathrm{Ui}=0,17$ correspondente a umidade final de 0,84 b.s. Ao mudarmos a 
espessura para $0,75 \mathrm{~cm}$ (Figura $3(D)$ ), os valores são também muito próximos: tempo de secagem 240 min. e a relação $\mathrm{Uo} / \mathrm{Ui}=0,17$ correspondente a umidade final de 0,84 b.s.

Sendo assim, os dados apontam para comportamento cinético similar para as condições operacionais utilizadas. Em todos os casos mostrados, a maior diferença observada diz respeito ao tempo necessário para o processo atingir umidade constante. Observa-se que quanto maior a velocidade do ar de secagem e menor a espessura da torta do material, as resistências difusionais e convectivas são minimizadas, facilitando a transferência de massa e calor do material para o ar, favorecendo dessa forma, a redução do tempo de secagem. Essa observação também foi relatada por Vashisth et al. (2011) e é explicada pela maior taxa de evaporação que acontece em condições de menor espessura e maior velocidade de ar.

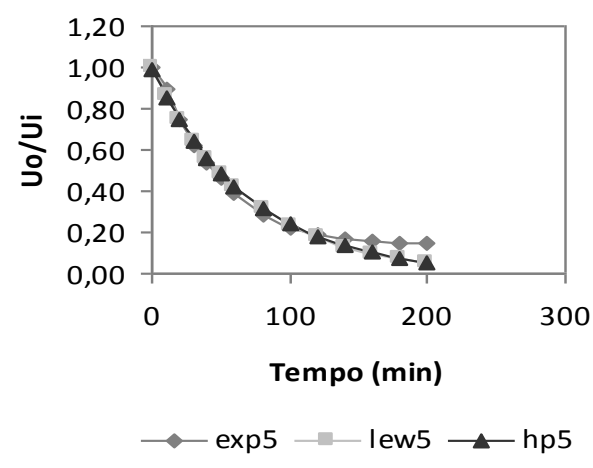

(E)

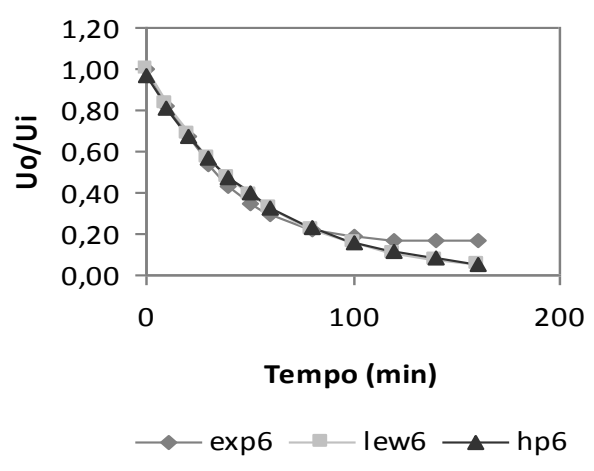

$(\mathrm{F})$

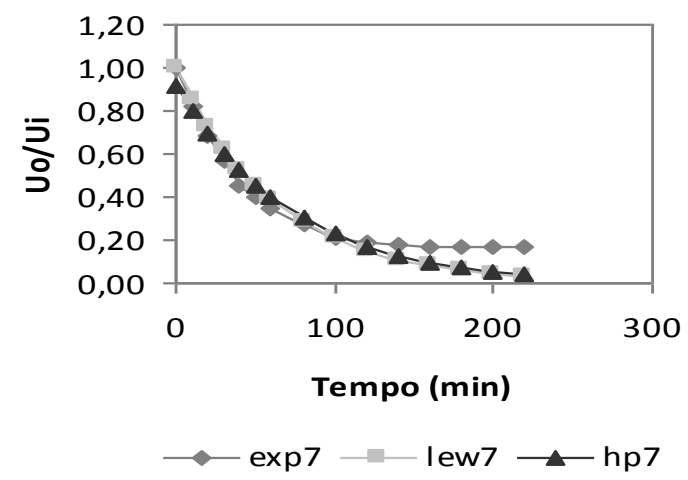

(G)

Figura 4 - Secagem do resíduo da acerola em secador de bandeja à temperatura de $70^{\circ} \mathrm{C}$, velocidade $5 \mathrm{~m} / \mathrm{s} \mathrm{e}$ espessura $0,62 \mathrm{~cm},(E) 1^{\circ}$ experimento, (F) $5^{\circ}$ experimento, (G) $9^{\circ}$ experimento. Legenda: (exp) valores experimentais, (lew) resultados ajustados ao modelo de Lewis e (hp) modelo de Henderson e Pabis.

Na Figura 4 (E), (F) e (G) estão apresentados os experimentos nas condições operacionais de temperatura de $70^{\circ} \mathrm{C}$, velocidade do ar de secagem de $5,0 \mathrm{~m} / \mathrm{s}$ e espessura da torta do material utilizado de $0,62 \mathrm{~cm}$, que constituem os experimentos de repetição do ponto central do planejamento experimental. Através das curvas é possível visualizar que nos 50 minutos iniciais, a redução de umidade do material varia linearmente, o que caracteriza o período de taxa constante. 


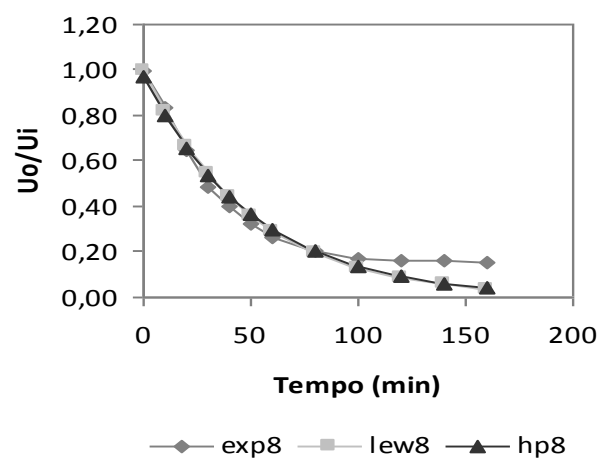

$(\mathrm{H})$

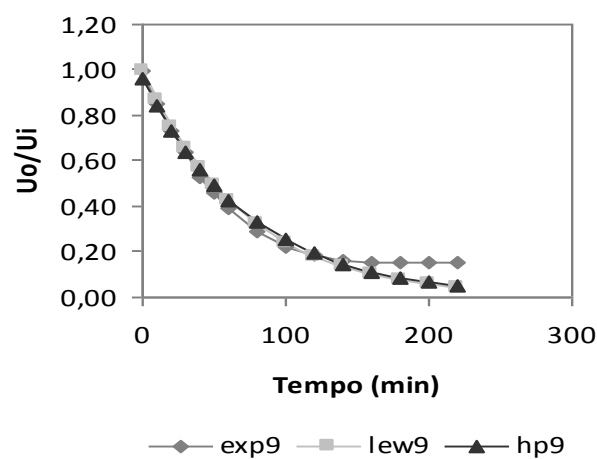

(I)

Figura 5 - Secagem do resíduo da acerola em secador de bandeja à temperatura de $80^{\circ} \mathrm{C}$ e velocidade de $4 \mathrm{~m} / \mathrm{s}$, (H) espessura de $0,50 \mathrm{~cm}$ e (I) espessura de $0,75 \mathrm{~cm}$. Legenda: (exp) valores experimentais, (lew) resultados ajustados ao modelo de Lewis e (hp) modelo de Henderson e Pabis.

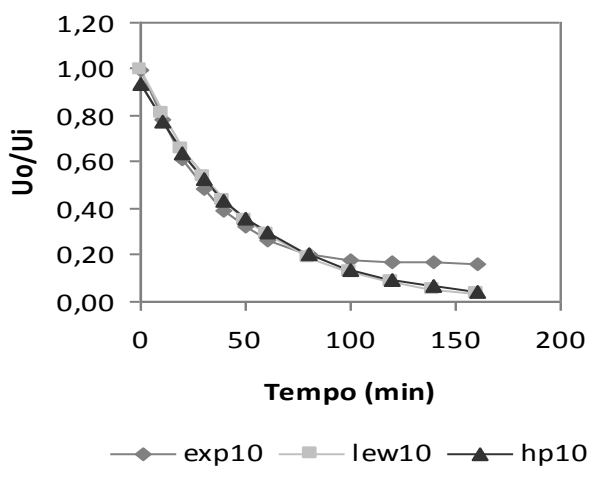

(J)

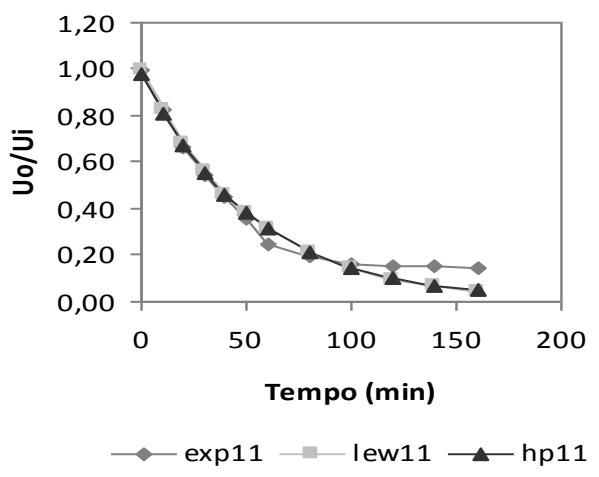

(K)

Figura 6 - Secagem do resíduo da acerola em secador de bandeja à temperatura de $80^{\circ} \mathrm{C}$ e velocidade de $6 \mathrm{~m} / \mathrm{s}$, (J) espessura de $0,50 \mathrm{~cm}$ e (K) espessura de $0,75 \mathrm{~cm}$. Legenda: (exp) valores experimentais, (lew) resultados ajustados ao modelo de Lewis e (hp) modelo de Henderson e Pabis.

Nas Figuras $5(\mathrm{H})$ e (I) e 6 (J) e (K) são apresentados os dados da secagem, sob condições de temperatura de $80^{\circ} \mathrm{C}$, velocidade do ar de secagem de 4,0 e $6,0 \mathrm{~m} / \mathrm{s}$ e espessura da torta do material utilizado de 0,5 e $0,75 \mathrm{~cm}$.

Verifica-se que, nas condições operacionais da Figura $5(\mathrm{H})$ com espessura de $0,5 \mathrm{~cm}$, o tempo de secagem foi de 160 minutos e a relação $\mathrm{Uo} / \mathrm{Ui}=0,16$ corresponde à umidade final de 0,86 b.s. Nas mesmas condições de temperatura e velocidade do ar, mas com maior espessura, $0,75 \mathrm{~cm}$ (Figura $5(\mathrm{I})$ ), o tempo de secagem sobe para 220 minutos e a relação Uo/Ui $=0,15$ corresponde à umidade final de 0,85 b.s.

Ao aumentar-se a velocidade do ar de secagem para $6 \mathrm{~m} / \mathrm{s}$, mantendo-se a temperatura de $80^{\circ} \mathrm{C}$ e utilizando espessura de $0,5 \mathrm{~cm}$ (Figura $6(\mathrm{~J})$ ), o tempo de secagem foi de 160 minutos e a relação $\mathrm{Uo} / \mathrm{Ui}=0,17$ correspondente a umidade final de 0,86 b.s. Nesse caso, ao variarmos a espessura para 0,75 cm (Figura $6(\mathrm{~K})$ ), o tempo de secagem se manteve em 160 minutos e a relação $\mathrm{Uo} / \mathrm{Ui}=0,15$ correspondente a umidade final de 0,87 b.s.

Nesse caso, o tempo de secagem sofreu influência da espessura da camada de material apenas nas condições de velocidade $4 \mathrm{~m} / \mathrm{s}$. Para velocidade de $6 \mathrm{~m} / \mathrm{s}$ a espessura da torta não 
influenciou o tempo de secagem de maneira que, a temperatura foi o parâmetro determinante no processo de secagem.

O formato das curvas apresentadas (Figuras 4, 5 e 6) permite inferir que, em todos os experimentos, a velocidade de secagem é maior no início do processo e diminui ao longo do processo. Este comportamento já esperado está de acordo com Park et al. (2001) e outros pesquisadores da área de secagem de alimentos.

Nas Tabelas 1 e 2 estão apresentados, respectivamente, os resultados dos ajustes dos modelos de Lewis e Henderson e Pabis aos dados experimentais. Além disso, também são mostrados os valores da constante de velocidade $(k)$, do coeficiente de correlação $\left(R^{2}\right)$, do quiquadrado reduzido $\left(\chi^{2}\right)$, do erro médio quadrático (RMSE) e uma estimativa de erros em percentual (\%E).

Tabela 1 - Parâmetros do modelo de Lewis.

\begin{tabular}{|c|c|c|c|c|c|c|}
\hline \multirow{2}{*}{$\begin{array}{c}\text { TEMPERATURA } \\
\left({ }^{\circ} \mathrm{C}\right)\end{array}$} & \multirow{2}{*}{ EXP } & \multicolumn{5}{|c|}{ PARÂMETROS - LEWIS } \\
\cline { 2 - 7 } & & $\mathrm{k}\left(\mathrm{min}^{-1}\right)$ & $\mathrm{R}^{2}$ & $\chi^{2}$ & $\mathrm{RMSE}$ & $\% \mathrm{E}$ \\
\hline \multirow{4}{*}{60} & 1 & $\mathbf{0 , 0 1 4 5}$ & $\mathbf{0 , 9 7 8 8}$ & $\mathbf{0 , 0 0 3 2}$ & $\mathbf{0 , 0 5 4 4}$ & $\mathbf{1 8 , 7 6}$ \\
\cline { 2 - 7 } & 2 & $\mathbf{0 , 0 1 0 3}$ & $\mathbf{0 , 9 8 7 0}$ & $\mathbf{0 , 0 0 2 0}$ & $\mathbf{0 , 0 4 3 7}$ & $\mathbf{1 5 , 0 1}$ \\
\cline { 2 - 7 } & 3 & $\mathbf{0 , 0 1 7 5}$ & $\mathbf{0 , 9 6 9 3}$ & $\mathbf{0 , 0 0 4 9}$ & $\mathbf{0 , 0 6 7 3}$ & $\mathbf{2 3 , 5 5}$ \\
\cline { 2 - 7 } & 4 & $\mathbf{0 , 0 1 2 5}$ & $\mathbf{0 , 9 7 5 1}$ & $\mathbf{0 , 0 0 4 0}$ & $\mathbf{0 , 0 6 1 3}$ & $\mathbf{2 1 , 8 6}$ \\
\hline \multirow{4}{*}{70} & 5 & $\mathbf{0 , 0 1 4 5}$ & $\mathbf{0 , 9 9 0 9}$ & $\mathbf{0 , 0 0 1 9}$ & $\mathbf{0 , 0 4 2 2}$ & $\mathbf{1 5 , 8 7}$ \\
\cline { 2 - 7 } & 6 & $\mathbf{0 , 0 1 8 7}$ & $\mathbf{0 , 9 8 8 1}$ & $\mathbf{0 , 0 0 3 0}$ & $\mathbf{0 , 0 5 2 7}$ & $\mathbf{1 8 , 6 1}$ \\
\cline { 2 - 7 } & 7 & $\mathbf{0 , 0 1 5 8}$ & $\mathbf{0 , 9 6 8 0}$ & $\mathbf{0 , 0 0 5 7}$ & $\mathbf{0 , 0 7 2 8}$ & $\mathbf{2 7 , 3 0}$ \\
\hline \multirow{5}{*}{80} & 8 & $\mathbf{0 , 0 2 0 4}$ & $\mathbf{0 , 9 7 8 1}$ & $\mathbf{0 , 0 0 3 6}$ & $\mathbf{0 , 0 5 7 3}$ & $\mathbf{2 1 , 8 9}$ \\
\cline { 2 - 7 } & 9 & $\mathbf{0 , 0 1 4 2}$ & $\mathbf{0 , 9 8 4 9}$ & $\mathbf{0 , 0 0 2 4}$ & $\mathbf{0 , 0 4 7 8}$ & $\mathbf{1 8 , 6 6}$ \\
\cline { 2 - 7 } & 10 & $\mathbf{0 , 0 2 0 9}$ & $\mathbf{0 , 9 6 9 6}$ & $\mathbf{0 , 0 0 4 5}$ & $\mathbf{0 , 0 6 4 4}$ & $\mathbf{2 4 , 2 1}$ \\
\cline { 2 - 7 } & 11 & $\mathbf{0 , 0 1 9 4}$ & $\mathbf{0 , 9 8 5 9}$ & $\mathbf{0 , 0 0 2 4}$ & $\mathbf{0 , 0 4 7 0}$ & $\mathbf{1 8 , 6 2}$ \\
\hline
\end{tabular}

Tabela 2 - Parâmetros do modelo de Henderson e Pabis.

\begin{tabular}{|c|c|c|c|c|c|c|c|}
\hline \multirow{2}{*}{$\begin{array}{c}\text { TEMPERATURA } \\
\left({ }^{\circ} \mathrm{C}\right)\end{array}$} & \multirow{2}{*}{ EXP } & \multicolumn{5}{|c|}{ PARÂMETROS - HENDERSON E PABIS } \\
\cline { 2 - 8 } & & $\mathrm{a}$ & $\mathrm{k}\left(\mathrm{min}^{-1}\right)$ & $\mathrm{R}^{2}$ & $\chi^{2}$ & $\mathrm{RMSE}$ & $\% \mathrm{E}$ \\
\hline \multirow{4}{*}{60} & 1 & $\mathbf{0 , 9 4 1 5}$ & $\mathbf{0 , 0 1 3 3}$ & $\mathbf{0 , 9 8 2 2}$ & $\mathbf{0 , 0 0 2 9}$ & $\mathbf{0 , 0 4 9 9}$ & $\mathbf{1 6 , 8 3}$ \\
\cline { 2 - 8 } & 2 & $\mathbf{0 , 9 5 0 6}$ & $\mathbf{0 , 0 0 9 6}$ & $\mathbf{0 , 9 8 9 5}$ & $\mathbf{0 , 0 0 1 7}$ & $\mathbf{0 , 0 3 9 3}$ & $\mathbf{1 3 , 1 6}$ \\
\cline { 2 - 8 } & 3 & $\mathbf{0 , 9 0 9 6}$ & $\mathbf{0 , 0 1 5 3}$ & $\mathbf{0 , 9 7 6 6}$ & $\mathbf{0 , 0 0 4 0}$ & $\mathbf{0 , 0 5 8 1}$ & $\mathbf{1 9 , 3 8}$ \\
\cline { 2 - 8 } & 4 & $\mathbf{0 , 9 2 5 3}$ & $\mathbf{0 , 0 1 1 2}$ & $\mathbf{0 , 9 8 0 1}$ & $\mathbf{0 , 0 0 3 3}$ & $\mathbf{0 , 0 5 4 1}$ & $\mathbf{1 8 , 3 4}$ \\
\hline \multirow{4}{*}{70} & 5 & $\mathbf{0 , 9 8 8 9}$ & $\mathbf{0 , 0 1 4 3}$ & $\mathbf{0 , 9 9 1 0}$ & $\mathbf{0 , 0 0 2 0}$ & $\mathbf{0 , 0 4 1 8}$ & $\mathbf{1 5 , 6 6}$ \\
\cline { 2 - 8 } & 6 & $\mathbf{0 , 9 6 7 9}$ & $\mathbf{0 , 0 1 7 9}$ & $\mathbf{0 , 9 8 8 7}$ & $\mathbf{0 , 0 0 3 1}$ & $\mathbf{0 , 0 5 0 8}$ & $\mathbf{1 7 , 8 9}$ \\
\cline { 2 - 8 } & 7 & $\mathbf{0 , 9 1 9 2}$ & $\mathbf{0 , 0 1 4 0}$ & $\mathbf{0 , 9 7 2 7}$ & $\mathbf{0 , 0 0 5 1}$ & $\mathbf{0 , 0 6 6 8}$ & $\mathbf{2 4 , 2 1}$ \\
\hline \multirow{5}{*}{80} & 8 & $\mathbf{0 , 9 7 0 4}$ & $\mathbf{0 , 0 1 9 6}$ & $\mathbf{0 , 9 7 8 8}$ & $\mathbf{0 , 0 0 3 8}$ & $\mathbf{0 , 0 5 6 3}$ & $\mathbf{2 1 , 1 2}$ \\
\cline { 2 - 8 } & 9 & $\mathbf{0 , 9 5 8 8}$ & $\mathbf{0 , 0 1 3 4}$ & $\mathbf{0 , 9 8 6 4}$ & $\mathbf{0 , 0 0 2 4}$ & $\mathbf{0 , 0 4 5 3}$ & $\mathbf{1 7 , 4 7}$ \\
\cline { 2 - 8 } & 10 & $\mathbf{0 , 9 3 7 8}$ & $\mathbf{0 , 0 1 9 1}$ & $\mathbf{0 , 9 7 3 2}$ & $\mathbf{0 , 0 0 4 4}$ & $\mathbf{0 , 0 6 0 5}$ & $\mathbf{2 1 , 8 3}$ \\
\cline { 2 - 8 } & 11 & $\mathbf{0 , 9 7 9 1}$ & $\mathbf{0 , 0 1 8 9}$ & $\mathbf{0 , 9 8 6 2}$ & $\mathbf{0 , 0 0 2 6}$ & $\mathbf{0 , 0 4 6 4}$ & $\mathbf{1 8 , 2 7}$ \\
\hline
\end{tabular}


Os resultados obtidos demonstram que os modelos Lewis e Henderson e Pabis descreveram satisfatoriamente o comportamento cinético do processo de secagem para as condições de operação trabalhadas. O coeficiente de regressão médio, $R^{2}$, para todos os experimentos foi maior ou igual a 0,98, independente da velocidade do ar de secagem e da espessura da torta, para os dois modelos. A constante de velocidade média para o modelo de Lewis foi de 0,0145 $\mathrm{min}^{-1}$ e para o modelo de Henderson e Pabis foi 0,015 $\mathrm{min}^{-1}$. Porém, considerando a estimativa dos erros, verifica-se que o modelo de Henderson e Pabis foi o que melhor se ajustou aos dados de secagem do resíduo de acerola.

\section{CONCLUSÃO}

Observou-se que a secagem ocorreu predominantemente em periodo de taxa decrescente, a partir de uma razão de umidade de aproximadamente $60 \%$ até uma razão de umidade em torno de $20 \%$. Em todos os experimentos notou-se, através dos gráficos, que os modelos utilizados descreveram satisfatoriamente o comportamento da secagem até o segundo período de taxa decrescente, onde a partir de então ambos apresentam desvios consideráveis. De modo geral, analisando-se os coeficientes estatísticos, conclui-se que o modelo de Handerson e Pabis ajustou melhor os dados experimentais em relação ao modelo de Lewis.

\section{AGRADECIMENTOS}

Os autores agradecem ao CNPq pelo apoio financeiro dado ao presente trabalho, através da concessão de bolsas de estudo.

\section{REFERÊNCIAS BIBLIOGRÁFICAS}

1. ALMEIDA, M. M. B; SOUSA, P. H. M.; ARRIAGA, A. M. C.; PRADO, G. M.; MAGALHÃES, C. E. C.; MAIA, G. A., LEMOS, T. L G. Bioactive compounds and antioxidant activity of fresh exotic fruits from northeastern Brazil. Food Research International, v.44, p. 2155-2159, 2011.

2. BRUNINI, M. A.; MACEDO, N. B.; COELHO, C. V.; SIQUEIRA, G. F. Caracterização física e química de acerolas provenientes de diferentes regiões de cultivo. Rev. Bras. Frutic., v. 26, n. 3, p. 486-489, 2004.

3. DEMBITSKY, V.; POOVARODOM, S.; LEONTOWICZ, H.; LEONTOWICZ, M.; VEARASILP, S.; TRAKHTENBERG, S.; GORINSTEIN, S. The multiple nutrition properties of some exotic fruits: biological activity and active metabolites. Food Research International, v. 44, p. 16711701, 2011.

4. FREIRE, L. A.C. Montagem e operação de um secador pneumático tipo flash. 2011. 89f. Dissertação (Mestrado em Engenharia Química) - Centro de Tecnologia, Departamento de Engenharia Química, Programa de Pós-Graduação em Engenharia Química, Universidade Federal do Rio Grande do Norte, Natal.

5. JESUS, M. F.; SCARANTO, V. L.; JALALI, V. R. R.; SILVA, G. F. Produção de passas de acerola em secador de bandeja. Revista Brasileira de Produtos Agroindustriais, Campina Grande, v.5, n.1, p.81-87, 2003.

6. LENART, A. Osmo-convective drying of fruits and vegetables: technology and application. 
Drying Technology, v. 14, n.2, p. 391 - 413. 1996.

7. MARQUES, L.; FERREIRA, M.; FREIRE, J. Freeze-drying of acerola (Malpighiaglabra L.), Chemical Engineering and Processing: Process Intensification, v. 46, n. 5, p. 451-457, 2007.

8. MARQUES, L.; PRADO, M.; FREIRE, J. Rehydration characteristics of freeze-dried tropical fruits. LWT-Food Science and Technology, v. 42, p. 1232-1237, 2009.

9. MEZADRI, T. et al. El fruto de la acerola: composición, características productivas e importancia económica. Archivos Latinoamericanos de Nutrición, v.56, p.101-109, 2006.

10. MEZADRI, T.; VILLAÑO, D.; FERNÁNDEZ-PACHÓN, M.;GARCÍA-PARRILLA, M.; TRONCOSO.Antioxidant compounds and antioxidant activity in acerola (Malpighiaemarginata DC.) fruits and derivatives. Journal of Food Composition and Analysis, v.21, n.4, p. 282-290, 2008.

11. MOREIRA. G. E. G.; COSTA, M. G. M.; SOUZA, A. C. R.; BRITO, E. S. ; MEDEIROS, M. F. D.; AZEREDO. H. M.C. Physical properties of spray dried acerolapomaceextract as affected by temperature and drying aids. Food Science and Technology, v. 42, p. 641-645, 2009.

12. PANCHARIYA, P.C.; POPOVIC, D.; SHARMA, A.L. Thin layer modelling of black tea drying process. Journal of Food Engineering v.52, p. 349-357, 2002.

13. PARK, K. J.; YADO, M. K. M.; BROD, F. P. R. Estudo de secagem de pêraBartlet (Pyrussp) em fatias. Ciência e Tecnologia de Alimentos, Campinas, v.2, n.3, 2001.

14. ROSSO, V.; MERCADANTE, A. Carotenoid composition of two Brazilian genotypes of acerola (Malpighiapunicifolia L.) from two harvests. Food Research International, v.38, n. 8-9, p. 1073-1077, 2005.

15. ROSSO, V. V.DE; MERCADANTE, A.Z. The high ascorbic acid content is the main cause of the low stability of anthocyanin extracts from acerola. Journal of Agricultural and Food Chemistry, v. 103, p.935-943, 2007.

16. ROSSO, V. V. DE; HILLEBRANDB, S.; MONTILLAB, E. C.; BOBBIO, F.O.; WINTERHALTERB, P.; MERCADANTE, A.Z. Determination of anthocyanins from acerola(Malpighiaemarginata DC.) and açai (Euterpeoleracea Mart.) by HPLC-PDAand Analysis, v. 21, p. 291-299, 2008.

17. SANTOS,C. T.; BONOMO, R. F.; CHAVES, M. A.; FONTAN, R. C. I.; BONOMO, P.Cinética e modelagem da secagem de carambola (Averrhoa carambolaL.) em secador de bandeja. Journal Acta Scientiarum: Technology, Maringá, v. 32, n. 3, p. 309-313, 2010.

18. VASHISTH, T.; SINGH, R. K.; PEGG, R. B. Effects of drying on the phenolics content and antioxidant activityof muscadine pomace. LWT - Food Sci. Technol., 44: 1649-1657, 2011. 